\title{
Comparison of the Glycerol Monostearate and Polyglycerol Stearate Oleogels: Effects of Amphiphile Addition
}

\author{
Emin YILMAZ ${ }^{1}$ and Eda KESKIN USLU ${ }^{1}$ \\ ${ }^{1}$ Çanakkale Onsekiz Mart University
}

April 28, 2020

\begin{abstract}
This study aimed to evaluate oleogels produced with Glycerol Monostearate (GMS), Polyglycerol Stearate (PGS), and their combinations with selected amphiphiles (Pluronic F68, Span 80, and Tween 60). The oleogels were prepared with oil: organogelator: amphiphile ratio of 87: 10: $3(\mathrm{w} / \mathrm{w})$. Minimum gelation concentration, gelation time, oil binding capacity, instrumental color, thermal properties, microstructure, X-ray diffraction, and rheological analyses were completed to characterize and compare the samples. Results indicated that the addition of amphiphiles reduced minimum gelation concentration, increased crystals size, changed color values, and reduced melting temperatures. Further, amphiphile addition yielded more stable, but softer textures. All samples were thermoreversible and thixotropic. Data for food-grade PGS oleogels were provided for the first time in literature. Results showed that PGS oleogels were as good as GMS oleogels in most properties. The prepared oleogels could be used in food product applications.
\end{abstract}

\section{Introduction}

Glycerol monostearate (GMS) is a mono-acyl ester of stearic acid with glycerol alcohol. It generally exists as enantiomeric pair of 1-glycerol monostearate and 2-glycerol monostearate. It is a white, odorless and sweet-tasting, hygroscopic flaky powder with a melting range of $73-81^{\circ} \mathrm{C}$. It is used as a thickening, emulsifying, anticaking, body forming, antistaling and preservative additive in the food industry. It is also used in cosmetic and pharmaceutical products (Anonymous 2020a; Anonymous 2020b). Polyglycerol stearate (PGS) is another ester of stearic acid with the polyglycerol molecule. Polyglycerols are, in fact, oligomers of glycerol. The nominal degree of polymerization and distribution of molecular species defines a specific polyglycerol ester. Usually, $70 \%$ of the polyglycerol moiety is composed of di-, tri- and tetraglycerols. Various types of fatty acid esters could be prepared with glycerol oligomers. Most PGS products are white to yellow colored, very viscous or plastic solids, soluble freely in oil and dispersible in water with a melting range of $55-65^{\circ} \mathrm{C}$. Triglyceryl esters of various fatty acids were shown to gelate liquid oils (Nakajima et al. 2012; Anonymous 2020c).

Oleogels or lipogels are semisolid materials made of an organogelator molecule and an edible liquid oil. The organogelators are the molecules which could create networks in liquid oil to entrap/immobilize or to gel it. There are numerous kinds of organogelators have already been used to prepare various oleogels, but the need for safe, efficient, low cost, abundant organogelators is still a driving force for new researches (Co and Marangoni 2012; Patel 2018a, b).

Literature for applications of GMS and PGS as organogelators for edible oleogel preparation are rather limited. In an early study (Sánchez et al. 2011), sorbitan and glyceryl monostearate were used to prepare oleogels with various liquid oils as green lubricants. Results showed that GMS oleogels were stronger than those of the sorbitan monostearate oleogels. Another study (Wang and Marangoni 2015) utilized GMS and sodium stearoyl lactylate (SSL) to prepare emulsion gels with added xanthan gum. They showed that 
the addition of xanthan gum to the GMS/SSL mixture resulted in enhanced stability of the $\alpha$-gel phase. Later the same group (Wang et al. 2016) investigated the effect of palm stearin addition to the glycerol monostearate-structured emulsions. Palm stearin addition changed the melting profile, and enhanced storage stability at refrigerator temperature, but destabilized the emulsion at room temperature. Structured reverse lyotropic liquid crystals (LLC) of glycerol monooleate (GMO), decane, water, and glycerol were shown to create emulsion oleogels (Cegla-Nemirovsky et al. 2015). These limited number of studies with GMS as organogelator were made on emulsion gels, but in this study, the GMS and its combinations with some selected amphiphiles were used as direct organogelator agents to structure edible liquid oil.

Di-, tri-, and tetra-fatty acid esters of linear glycerol oligomers (2-10 glycerol) were synthesized and evaluated for oleogel formation ability (Nakajima et al. 2012). Since this study was published in the Japanese language, we could only obtained the abstract. They indicated that triglyceryl esters of alkyl chains with suitable length created stable oleogels. No other study was found in the literature with PGS as organogelator for edible oleogels.

Some studies were indicated the stabilizing effects of some added amphiphiles for stearic acid oleogels (Uvanesh et al. 2016a, b). In one of them (Uvanesh et al. 2016a), the addition of Span 60 into the stearic acid oleogel changed the crystal shape from plate-like structure to branched architecture. Also, nucleation induction time was shortened, and physical stability was increased. It was suggested that Span 60 could be used to alter the kinetics of the crystallization, crystal habit and crystal structure of stearic acid oleogels. In the other study (Uvanesh et al. 2016b), Tween 20 was investigated for the same purpose in stearate oleogels. The addition enhanced stability of B polymorph and leaf-like structure. Further, a delay in induction time for nucleation and the increase in crystal ordered arrangements were observed. Hence, the effects of some added amphiphiles for the GMS and PGS oleogels would also deserve investigation.

In the present study, glycerol monostearate (GMS) and polyglycerol stearate (PGS) were used as direct organogelators to prepare sunflower oil oleogels. Further, the effects of adding selected amphiphiles on the oleogel properties were investigated. After preparing the oleogels, characterization studies for main physical properties, microstructure, thermal behavior and rheological properties were provided. The aim was to determine suitable organogel and amphiphile combinations for edible oleogel applications.

\section{Materials and Methods}

\section{Materials}

Refined-winterized sunflower oil (Trakya Birlik Oil Co., Tekirdağ, Turkey) was purchased and used as liquid oil to prepare the oleogels. Purified powder glycerol monostearate (GMS) was purchased from Alfa Aesar GmbH \& Co KG (Karlsruhe, Germany). Creamish powder of polyglycerol stearate (Finamul PGE 2000) was purchased from Fine Organics Inc. (Mumbai, India). The amphiphiles, Pluronic F68 (10\% poloxamer 188 solutions), Span 20, Span 60, Span 80, Tween 40 and Tween 60 were bought from Sigma-Aldrich Chemie GmbH (Taufkirchen, Germany). Similarly, Span 65, Tween 20 and Tween 80 were purchased from Merck Schuchardt OHG (Hohenbrunn, Germany). Soy lecithin was bought from Kimbiotek Kimyevi Maddeler San. Co. (Istanbul, Turkey). All other chemicals and solvents used were of analytical grade and were purchased from Sigma Chem. Co. (St. Louis, MO, USA) or Merck (Darmstadt, Germany).

\section{Preparation of theOleogels}

The minimum gel concentrations $\left(\mathrm{C}^{*}\right)$ of the GMS and PGS alone were determined first. The oil: organogelator (GMS or PGS) mixtures from 99:1 to 90:10 (w/w) were prepared in tubes and heated in a water bath at $90^{\circ} \mathrm{C}$ for full melting. The tubes vortexed vigorously for homogeneity. The mixtures were taken to ambient temperature $\left(20 \pm 3^{\circ} \mathrm{C}\right)$ and stayed overnight at ambient temperature. The next day, the tubes were examined by eye for any flow by tilting $90^{\circ}$, and the tubes with no flow were selected with their organogelator addition level as the $\mathrm{C}^{*}$ value. Consequently, the $\mathrm{C}^{*}$ values of GMS and PGS were determined as $6.0 \%$ and 5.0\%, respectively.

The next step was to select the amphiphiles which able to enhance oleogel stability. To do this, organogelator 
addition levels of one unit below their $\mathrm{C}^{*}$ values were selected (5.0\% for GMS and $4.0 \%$ for PGS). Ten tubes for each organogelator were prepared and $3 \%(\mathrm{w} / \mathrm{w})$ of each selected amphiphiles (Pluronic F68, Span 20, Span 60, Span 65, Span 80, Tween 20, Tween 40, Tween 60, Tween 80, and soy lecithin) were added. The were tubes melted and prepared in a water bath in the same way. The next day, the tubes without flow (stable gels) were selected. By this procedure, the amphiphiles, which created stable oleogels, were actually selected, since at one unit lower level of organogelator addition, none of the oleogels were stable by themselves. The Pluronic F68 and Span 80 for GMS oleogel, and Pluronic F68 and Tween 60 for PGS were selected as the stability-enhancing amphiphiles.

After selecting the amphiphiles, the oleogel formulations were prepared at oil: organogelator: amphiphile (87: 10: $3, \mathrm{w} / \mathrm{w})$ ratios. The control samples were oil: organogelator $(90: 10, \mathrm{w} / \mathrm{w})$ samples. The organogelator addition level $(10 \%)$ well above the $\mathrm{C}^{*}$ values were selected to get rather strong gels to make meaningful and easy comparisons. The formulations of the six different oleogels prepared and evaluated in this study were shown in Table 1. The prepared oleogel samples could be observed in Fig. 1. All further analyses were completed on the samples prepared with the given formulae in Table 1. Further, the abbreviated samples names provided in Table 1 were used throughout the paper.

\section{Physical Properties of the Oleogels}

The gelation time (GT) is defined as the time required for an oleogel to fully solidify at a given melting and cooling temperature. The prepared oleogels were first fully melted in a water bath at $90^{\circ} \mathrm{C}$ for $30 \mathrm{~min}$. Then, taken to ambient temperature, and the timer was started. Once the oleogels solidified, the time elapsed was read as the gelation time in minutes (Yilmaz and Ögü̈tcü 2014).

Oil binding capacity $(\mathrm{OBC})$ is the parameter indicating what a known amount of organogelator could entrap percent of liquid oil. The oleogel samples were melted $\left(90^{\circ} \mathrm{C}\right.$ for $\left.30 \mathrm{~min}\right)$, and $1 \mathrm{ml}$ of each melted sample was placed into tared Eppendorf tubes. The tubes were stored at the refrigerator for $1 \mathrm{~h}$ for full gel formation. Finally, the tubes were weighed and centrifuged (10.000 rpm for $15 \mathrm{~min})$, before drainage of the released oil on the paper cloth. The tubes were weighed again, and the OBC was calculated gravimetrically (Yilmaz et al. 2015).

The color of the samples were assessed with a Minolta CR-400 (Konica Minolta Sensing, Osaka, Japan) colorimeter according to CIE standards, and the values of $\mathrm{L}, \mathrm{a}^{*}$ and $\mathrm{b}^{*}$ were recorded on several points over the samples (Yilmaz et al. 2015).

\section{Microstructural Properties of the Oleogels}

To observe the basic microstructural scenes, the polarized light microscopy images of the oleogels were recorded with an Olympus BX51 polarized light microscope (Olympus Optical Co., Ltd., Japan) equipped with a CCD color video camera (Canon) at room temperature (Yilmaz and Öğütcü 2014).

The polymorphic types of crystals formed in the oleogel samples were determined with a PANalytical Empyrean model (The Netherlands) X-ray diffractometer by following Cj 2-95 method (AOCS 1998). The oleogel samples were kept at ambient temperature overnight and loaded at ambient temperature to the sample holder of the instrument. The angular scans $(2 \vartheta)$ were performed from $2.0^{\circ}$ to $50^{\circ}$ by $2^{\circ} / \mathrm{min}$ scan rate under a $\mathrm{Cu}$ source X-ray tube $(\lambda=1.54056 \AA, 40 \mathrm{kV}$ and $40 \mathrm{~mA})$. Data analysis was accomplished with X'Pert HighScore Plus software (Malvern Panalytical Ltd., Royston, UK) (Yilmaz et al. 2015).

\section{Thermal Properties of the Oleogels}

The thermal parameters (crystallization and melting onset, peak temperatures and enthalpies) of the GMS, PGS and the six different oleogels prepared were assessed with a Perkin-Elmer 4000 Series Differential Scanning Calorimeter (DSC) (Groningen, The Netherlands). The instrument was previously calibrated with Indium and Zinc. Around $8 \mathrm{mg}$ of each sample was placed into the aluminum pans and sealed. The temperature program: heat samples from $20^{\circ} \mathrm{C}$ to $100^{\circ} \mathrm{C}$ by $10{ }^{\circ} \mathrm{C} / \mathrm{min}$; cool samples to $-30{ }^{\circ} \mathrm{C}$ by $10{ }^{\circ} \mathrm{C} / \mathrm{min}$ rate and keep $3 \mathrm{~min}$ at that temperature for full crystallization; and finally heat samples again to $100{ }^{\circ} \mathrm{C}$ by 
$5{ }^{\circ} \mathrm{C} / \mathrm{min}$ rate. The Pyris 1 Manager Software of the instrument were used for the calculations (Yilmaz et al. 2015).

\section{Rheological Properties of the Oleogels}

A DHR 2 rheometer (TA Instruments, USA) with a Peltier system $\left( \pm 0.1^{\circ} \mathrm{C}\right)$ under the lower plate donated with cross-hatched parallel plate geometry $(\varphi=40 \mathrm{~mm}$, gap $0.9 \pm 0.1 \mathrm{~mm})$ was used in all analyses. The samples were measured at $10^{\circ} \mathrm{C}$ to get a better observation for the rheological properties since the samples at $10{ }^{\circ} \mathrm{C}$ were very sufficiently stable. An amplitude sweep test with $0.01-100 \%$ strain and $1 \mathrm{~Hz}$ frequency was completed first to determine the linear viscoelastic region (LVR) for each sample. The LVR is defined as the region where a plateau for the storage $\left(G^{\prime}\right)$ ve loss $\left(G^{\prime \prime}\right)$ moduli from the stress sweeps must be observed, and all valid rheological assessments must be done within the LVR by definition (Mezger 2014).

The strain ranges of $0.016-0.232 \%$ and frequencies from 0.1 to $100 \mathrm{~Hz}$ were selected for frequency tests at 10 ${ }^{\circ} \mathrm{C}$. To evaluate the structural recovery ability of the oleogels under specific stresses applied, a time sweep test was completed. Three strain gradient regions were selected and applied to the samples at $10^{\circ} \mathrm{C}$ with $1 \mathrm{~Hz}$ frequency. The first region was applied for $180 \mathrm{~s}$ at each LVR determined to simulate standard force application. In the second region, strains well above each LVR determined were applied for $180 \mathrm{~s}$ to simulate the structural breakdown conditions. Finally, strains much lower than those of the LVR strains were applied for $900 \mathrm{~s}$ to simulate the structural recovery region.

Since all previous rheological measurements were completed at $10^{\circ} \mathrm{C}$, the effects of temperature on oleogel structure was a deserving observation, and a temperature ramp test was applied. The test was accomplished by heating the samples from $0^{\circ} \mathrm{C}$ to $80^{\circ} \mathrm{C}$ by $1^{\circ} \mathrm{C} /$ min rate at $1 \mathrm{~Hz}$ frequency within the LVR with $120 \mathrm{~s}$ soak time. All rheological analyses were repeated at least twice and average values were given.

\section{Statistical Analysis}

The six different oleogels were prepared at two different times as replicates, and each replicate production samples were analyzed for at least in duplicate and for some analyses in triplicate. The collected data were calculated as mean values with standard deviations. The Analysis of Variance (ANOVA) and Tukey's test was completed for means separation of the physical properties measured. The level of confidence was at least 95\%. Statistical analysis was performed with Minitab v.16.1 software (Minitab 2010).

\section{Results and Discussion}

\section{Physical Properties}

The organogelators used in this study were the GMS and PGS, and it would be helpful first to discuss their main properties and uses as food additives. GMS was an off-white powder with over $95 \%$ of monoglyceride ester, with a formula weight of 358.56 , and max iodine value of $6.0 \mathrm{gI}_{2} / 100 \mathrm{~g}$ sample. Its melting range was given as $57-74^{\circ} \mathrm{C}$. It was defined as soluble in hot organic solvents and hot water, slightly soluble in ethanol, and insoluble in aliphatic solvents. The application areas of GMS were specified as an emulsifier, resin lubricant, opacifier, emollient, bodying agent in a variety of cosmetic formulations for skincare and haircare. Further, it was used in food products as a thickening, anti-caking, foaming, anti-staling, and preservative agent (Anonymous 2020b). Similarly, PGS was a creamish powder with neutral odor and taste, with max $5.0 \mathrm{mg} \mathrm{KOH} / \mathrm{g}$ acid value, $130-150 \mathrm{mg} \mathrm{KOH} / \mathrm{g}$ saponification value, and 50-60 ${ }^{\circ} \mathrm{C}$ melting range. It was also mainly used as an emulsifier and whipping agent in food products (Anonymous 2020c). Clearly, both organogelators are legal food additives with known safety and legal status. Basically, they are high melting point emulsifiers. Hence, oleogel forming ability must arise from the junction zones formed at the interfaces of polymer chains and crystals of stearic acid within the molecules. In the prepared oleogels, the amphiphiles Pluronic F68, Span 80 and Tween 60 were also used as co-gelator or stability enhancing agents. The Pluronic F68 or Poloxamer 188 is a copolymer of polyoxyethylene and polyoxypropylene. It was prepared as $10 \%$ sterile, bio-reagent suitable for cell cultures. Its molecular weight was around 8350, and specified as a non-ionic biodetergent with 29.0 HLB number (Anonymous 2020d). Span 60 or Sorbitan monostearate is a non-ionic bio detergent with 430.63 molecular weight, $4.7 \mathrm{HLB}$ number, and $113{ }^{\circ} \mathrm{C}$ flash point (Anonymous 2020e). 
Similarly, Tween 60 or Polyoxyethylene sorbitan monostearate is a non-ionic detergent with 1309 molecular weight and 14.9 HLB number (Anonymous 2020f). Clearly, both the organogelators and the stabilizing agents used were mainly amphiphilic molecules with diverse molecular weight, solubility and HLB values, which determines their behavior in the liquid oil medium.

Some common physical properties of the prepared oleogels were measured and the findings were presented in Table 2. These oleogels were prepared according to the formulae given in Table 1 for better comparison purposes. There were slight differences among the samples for gelation time (GT) values. In both GMS and PGS oleogels, amphiphile addition enhanced GT compared to their controls. All oleogels were fully formed between 3.3 and 6.3 minutes. In fact, this duration could be perceived as quite short, since there was no external cooling during oleogel formation. In literature, quite diverse GT values from minutes to hours were reported. It was suggested that organogelator kind and addition level, oil type, presence and rate of cooling, preparation technique used and other factors determine the GT of oleogels. Shorter GT could be accepted as an advantage during actual uses for time-saving and speed for structural recovery (Dassanayake et al. 2011; Co and Marangoni 2012; Singh et al. 2017; Patel 2018a, b).

Oil binding capacity (OBC) specifies the liquid oil entrapment ability of the organogelators, and provides information about what percent of the liquid oil is being immobilized. The measured OBC values (Table 2) indicate that all oleogels had values above $99 \%$ without any significant difference among the samples. This is rather a very good finding showing the perfect efficiency of the organogeltors used. This result mostly concurs with the known literature (Patel 2018b).

The color of the oleogels were affected by the organogelator type as well as by the added amphiphiles. The highest L value (68.3) was measured in the GMS/F68-O sample, and the lowest value (46.9) was in the PGS/TW60-O sample (Table 2). L value indicates the level of brightness, and ranges between 0 (dark) to 100 (white) in the CIE measuring system (Pomeranz and Meloan 1994). Similarly, the a* value shows redness/greenness level, and as it goes on a positive direction, the level of redness enhances, and vice versa. All samples had some small level of green tones with the GMS/F68-O as the greenest sample. The b* value indicate yellowness/blueness value (positive values-yellow; negative values-blue). All GMS oleogels were yellow tones samples, and all PGS samples had slight blue tones, according to the color data (Table 2 ). The color of the liquid oil mainly constitutes color of an oleogel used to prepare it, but organogelators added and other additives used could significantly change the color tones. Since the same stock oil (refineswinterized sunflower oil) was used in all oleogels prepared, the measured color differences could be attributed to the organogelators and the amphiphiles used. The color of an oleogel could be essential when it was used in actual food products. Since each food has its respective color ranges in the eye of consumers, added oleogel shall not change the color of the food significantly. As could be observed (Fig. 1), all prepared oleogels in this study generally had light creamy-white colors, and could not create any difficulty in food formulations. This issue could be a limiting factor in the selection of different organogelators.

\section{Microstructural Properties}

Microstructural evaluation of an oleogel provides opportunity to understand how the liquid oil entrapped, which mechanism was involved during the immobilization and what was the structure resembles. First, the polarized light microscopy (PLM) images of the gels were provided in Fig. 2. In the PLM images, the lipid crystals appear as bright white scenes, and liquid oil is observed as the black backgrounds. Hence, the crude crystal morphology could be perceived by eye evaluation. As observed (Fig 2a), GMS-O represent the smaller and dense crowd of lipid particles. Once the amphiphiles Pluronic F68 added (Fig. 2b), the crystals get bigger, and when Span 80 added, the lipid crystals became large aggregates. A similar trend was evident in the amphiphiles added PGS oleogels (Fig. 2e, f), where lipid crystals became larger. It could be due to the conglomeration of the previously formed lipid crystals by the amphiphiles located at their surfaces. The effect of Span 60 on stearic acid oleogel structure was previously studied (Uvanesh et al. 2016a). The addition of Span 60 changed the stearate crystal shape from plate-like to branched architecture. Further, significant variations of the solute inside the oleogel have occurred. Formation of the branched-fiber structures was explained by the interfacial energy reduction in the nuclei due to the synergistic interactions amongst 
the gelator (stearic acid) and the co-gelator (Span 60) molecules. In another similar study (Uvanesh et al. 2016b), the effect of Tween 20 addition on the stearic acid oleogels was investigated. It was shown that Tween 20 addition enhanced polymorph B structure, reduced mobile fraction and delayed induction time of crystallization. In general, results in this study are in good agreement with the studies of Uvanesh et al. (2016a, b). Indeed, the GT of the oleogels were increased (Table 2), and the crystals enlarged to aggregate structures (Fig. 2). Beyond these, it was evident that the formation of oleogels was possible at one percent lower addition levels of the GMS and PGS with the addition of selected amphiphiles as presented with the $\mathrm{C}^{*}$ values provided in Table 1 . Overall, it seems clear that the addition of amphiphiles enhanced oleogel stability by increasing lipid particle sizes.

The X-ray diffraction patterns of the oleogels were presented in Fig. 3. The AOCS official method Cj 2-95 (AOCS 2012) identifies the peak at $\mathrm{d}=4.15 \AA$ as the $\alpha$ polymorphic form, two peaks at positions $\mathrm{d}=$ $3.8 \AA$ and $\mathrm{d}=4.2 \AA$ as the $\beta^{\prime}$ polymorphic form, and a peak appears at position $\mathrm{d}=4.6 \AA$ as the $\beta$ polymorphic form. Further, these d-spacings obtained from Bragg peak define the subcell within the crystal lattices. According to the method and patterns presented in Fig. 3, the GMS-O sample had peaks similar to $\beta^{\prime}$ polymorphic form. GMS/F68-O is more similar to $\beta$ polymorph, whereas GMS/SP80-O seems to include both $\beta$ and $\beta^{\prime}$ polymorphic forms. All three PGS oleogels showed patterns somewhat similar to $\beta$ polymorphic form. It is well known that $\alpha$ form includes the hexagonal crystal lattice, while $\beta^{\prime}$ polymorphic form identifies the orthorhombic, and $\beta$ polymorphic form identifies the triclinic subcell crystal lattices. Differences among the samples could be due to the added amphiphiles. The polymorphic crystal forms in commercial solid lipid products have said to have a paramount effect on product sensory quality and shelf-life. It was stated that $\beta^{\prime}$ polymorphic form represents very fine, homogeneous, creamy texture with a slippery, creamy mouth feel, and this polymorph is preferred in margarine, spreads and similar products. Sandy, coarse but very stable texture with harsh mouth feelings are mostly evident with $\beta$ type polymorph, and it is preferred for sugar confectionery and bakery shortenings (Chrysam 1996). As could be observed from Fig. 1, all oleogels prepared in this study seems rather very fine, creamy and homogeneous soft solids. Overall, the prepared oleogels could be suitable in food formulations utilizing semi-solid consistency fat, or in margarine, spreads and similar product productions.

\section{Thermal Properties}

The crystallization and melting onset, peak temperatures, and enthalpies of the organogelators GMS and PGS, and the six different oleogels prepared were assessed by DSC, and the results were summarized in Table 3. The GMS included two fractions with different thermal behavior; one fraction crystallizes at around $12.99^{\circ} \mathrm{C}$, and the other one at around $62.27^{\circ} \mathrm{C}$. These two fractions showed melting peak temperatures as 16.68 and $68.88{ }^{\circ} \mathrm{C}$, respectively. Obviously, the GMS used to include some isomeric forms or impurities. Contrarily, PGS had only one peak, it crystallizes at $49.53^{\circ} \mathrm{C}$ and melts at $58.39{ }^{\circ} \mathrm{C}$. The three oleogels made with GMS organogelator also had two fractions. The major fraction (with higher $\Delta \mathrm{H}$ value) presented certain behavior with added amphiphiles. While GMS-O sample had $56.34^{\circ} \mathrm{C}$ melting peak temperatures, the sample with added Pluronic F68 (GMS/F68-O) showed $55.83^{\circ} \mathrm{C}$, and the sample with added Span 80 (GMS/SP80$\mathrm{O})$ had $53.25^{\circ} \mathrm{C}$ peak melting temperatures. Clearly, added amphiphiles reduced melting temperatures. The same trend was not evident in the PGS oleogels. PGS-O had $37.92^{\circ} \mathrm{C}$ peak melting temperature, whereas PGS/F68-O and PGS/TW60-O had 41.67 and $41.45{ }^{\circ} \mathrm{C}$ peak melting temperatures, respectively (Table 3). It must be kept in mind that the oleogels with organogelator alone had $10 \%$ organogelator added, but samples with amphiphiles had $7 \%$ of the organogelators and $3 \%$ of the amphiphiles. Hence, different proportions and melting profiles of each added molecules could change the thermal behavior of the resulting oleogel. Overall, the crystallization and melting profiles of the oleogels prepared in this study seems quite acceptable for food applications compared to other known oleogels in the literature (Patel 2018a, b; Mattice and Marangoni 2018; Bot and Flöter 2018).

\section{Rheological Properties}

An amplitude sweep test was completed at first to determine the linear viscoelastic region (LVR) for each sample. The test was completed at $1 \mathrm{~Hz}$ frequency, $0.01-100 \%$ strain and $10{ }^{\circ} \mathrm{C}$. During the oscillatory 
test, the sinusoidal function was observed and the phase shift $(\delta)$ between $0^{\circ}$ and $45^{\circ}$ was maintained for all samples. The graphs of the amplitude sweep tests were presented in Fig. 4. These graphs show the oscillatory strain (\%) versus storage $\left(G^{\prime}\right)$ and loss $\left(G^{\prime \prime}\right)$ moduli changes. In these graphs, the LVR is observed as the plateau where storage modulus $\left(\mathrm{G}^{\prime}\right)$ and loss modulus $\left(\mathrm{G}^{\prime \prime}\right)$ behave like constant before deformation occurs to decrease both lines. It is important to determine the LVR of each sample, since all of the rheological measurements must be carried out within the LVR limits. LVR indicates the range within which the sample could be stressed without destroying its structure (Mezger, 2012). The LVR oscillatory strains were determined as 0.016, 0.058, 0.015\% for the GMS-O, GMS/F68-O, GMS/SP80-O, and 0.061, $0.156,0.232 \%$ for the PGS-O, PGS/F68-O, PGS/TW60-O, respectively. Within the LVR, all samples hold the $\mathrm{G}^{\prime}>\mathrm{G}^{\prime \prime}$ condition, which proves the exact gel-like structure of the samples. Since the storage modulus $\left(G^{\prime}\right)$ represents the elastic portions or describes the solid-like properties of the oleogel samples, it would be better to compare the $\mathrm{G}^{\prime}$ values of the samples within LVR to determine their relative strength. The $\mathrm{G}^{\prime}$ values of around 15.000, 14.000, and 500.000 Pa were read for GMS-O, GMS/F68-O, and GMS/SP80-O, respectively. Similarly, values of around 9.000, 8.000, and 17.000 Pa were observed for the PGS-O, PGS/F68$\mathrm{O}$, and PGS/TW60-O, respectively. Clearly, among all oleogel samples, the GMS/SP80-O was the strongest, and PGS/F68-O was the weakest samples. All samples reached the crossover point $\left(\mathrm{G}^{\prime}=\mathrm{G}^{\prime \prime}\right)$ at around 4-11\% oscillatory strain range (Fig. 4). Once a sample passes the crossover point, it lost its viscous portion and starts to flow like a fluid. Therefore, oscillatory strain values higher than crossover point values destroy samples gel structure, and precaution must be given during shear requiring processes.

The frequency sweep test results of the samples were given in Fig. 5. For each sample, the oscillatory strain determined at the LVR, angular frequencies between 1 and $1.000 \mathrm{rad} / \mathrm{s}$ were applied at $10^{\circ} \mathrm{C}$ to complete the frequency tests. Data presented in Fig. 5 shows the storage $\left(G^{\prime}\right)$ and loss $\left(G^{\prime \prime}\right)$ moduli, and complex viscosity $\left(\eta^{*}\right)$ values. As also evidenced from the amplitude sweep test results, all samples had $\mathrm{G}^{\prime}>\mathrm{G}^{\prime \prime}$ condition in the frequency sweep tests to prove that all were gel-like soft solids. It is well known that the storage modulus $\left(\mathrm{G}^{\prime}\right)$ of a sample indicates the elastic portion and represents the solid-like properties, while the loss modulus $\left(G^{\prime \prime}\right)$ indicates the viscous portion and represents the liquid-like properties. That's why a gel must hold the $\mathrm{G}^{\prime}>\mathrm{G}^{\prime \prime}$ condition is explained (Mezger 2014). Further, in all samples, the complex viscosity $\left(\eta^{*}\right)$ values were decreased as angular frequency gradually enhanced. This type of phenomena is called as shear thinning behavior, and previously observed in most plant wax oleogels (Mattice and Marangoni 2018; Patel 2018a). The $\mathrm{G}^{\prime}$ values of around $600.000 \mathrm{~Pa}, 120.000 \mathrm{~Pa}$, and 230.000 $\mathrm{Pa}$ were measured for GMS/SP80-O, GMS/F68-O, and GMS-O samples throughout the applied frequency range, respectively. Clearly, the addition of Span 80 increased gel strength, while the addition of Pluronic F68 decreased it, compared with the control sample. The very similar trend was evident for the PGS oleogels, in which PGS/TW60-O sample had around 500.000 Pa G' values, while PGS/F68-O had only around 6.000 Pa G' value. The control sample (PGS-O) had around 15.000 $\mathrm{Pa} \mathrm{G}^{\prime}$ value. Clearly, in both oleogel samples, the addition of Pluronic F68 amphiphile enhanced stability but decreased gel stiffness. The samples showed $\mathrm{G}^{\prime}$ : $\mathrm{G}^{\prime \prime}$ ratio of around 4:1 to 8:1, which indicate that the gels are flexible to a certain degree. Only the PGS/TW60-O sample had that ratio approaching to 10:1, which indicate a stiff and inflexible gel structure. Sometimes, the inverted ratio $\left(\mathrm{G}^{\prime \prime}\right.$ $\left./ G^{\prime}\right)$ or the loss factor $(\tan \delta)$ is used. Loss factor values lower than 0.1 indicate that $\left(\mathrm{G}^{\prime \prime} / \mathrm{G}^{\prime}[?] \quad 0.1\right.$ conditions) the gels are strong in nature. In the samples of GMS/SP80-O, GMS/F68-O, and GMS-O, the loss factors were around $0.16,0.25$, and 0.22 , indicating that all were rather weak gels. In fact, the GMS/SP80-O sample could be accepted as an almost strong gel. Similarly, the loss factors of PGS/TW60-O, PGS/F68-O, and PGS-O were around 0.1, 0.16 and 0.66, respectively. Consequently, the PGS/TW60-O sample could be accepted as a strong, and PGS/F68-O sample as an almost strong gel. Since all weak oleogels had loss factor values within the 0.2-0.3 range, they resist syneresis. Further, within the applied frequency range, none of the samples showed the crossover point $\left(G^{\prime}=G^{\prime \prime}\right)$, indicating that throughout the range all samples kept their viscous state.

The time sweep tests were also conducted for the oleogel samples (Fig. 6). To evaluate time-dependent viscoelastic behavior, shearing under dynamic mechanical conditions, that is shearing under constant amplitude and frequency were applied for each individual test intervals. Three-time domains with three different 
shearing options at $1 \mathrm{~Hz}$ and $10^{\circ} \mathrm{C}$ were used for these tests. The first region was lasted for $180 \mathrm{~s}$ with strains at LVR for each sample to simulate the resting conditions. Then strains well above LVR to simulate structural breakdown were applied for another $180 \mathrm{~s}$, and finally strains well below LVR strains were applied for $900 \mathrm{~s}$ to simulate the structural recovery region. The data obtained by the tests were shown in Fig. 6 . The changes in the complex shear modulus $\left(G^{*}\right)$ could be followed from the graphs. The $G^{*}$ represents entire viscoelastic behavior and divided into two portions; the storage modulus ( $\left.G^{\prime}\right)$ and the loss modulus (G"). In fact, it was possible two provide the $G^{\prime}$ and $G^{\prime \prime}$ in Fig. 6 , but it was too crowded with lines. Since $G^{*}$ could also show the structural changes, it was preferred to simultaneously observe 3 samples in each graph. In the first region simulating resting condition, the $\mathrm{G}^{*}$ values representing those measured in the frequency sweep tests were observed, respectively. In the second region, $G^{*}$ values decreased significantly as the applied force enhanced, and the structure is broken down, respectively. In this region, the gel state was lost, and the samples became more fluid-like. At the third region, the applied strains were well below LVR strains to let the gelled structure to recover, and in fact, it occurred in all samples, as evidenced from the enhancing $G^{*}$ values again to the level measured at the first stage. Clearly, structural reformation or recovery was present in all samples upon the reduction of the initial load applied to the samples. This behavior is common in gels and called thixotropic behavior. Thixotropy was previously observed in most wax oleogels (Patel 2016; Mattice and Marangoni 2018). In fact, oleogels with thixotropic recovery ability could be advantageous during food processing unit operations. Food processings like mixing, whipping, foaming etc. use certain forces, and the gel state could lose depending on the magnitude of the force. The gel state could recover upon the ceasing of the applied force. Hence, oleogels prepared in this study could be eligible for force requiring food processing unit operations.

Lastly, the effect of temperature change upon gel structure was studied by a temperature ramp test applied by increasing the temperature of the surrounding from $0{ }^{\circ} \mathrm{C}$ to $80^{\circ} \mathrm{C}$ under constant frequency and amplitude (Fig. 7). The changes in the storage modulus (G'), loss modulus (G"), and tan $\delta$ values through the heating ramp could be observed in Fig. 7 for each sample. In all samples, as temperature enhanced, both storage $\left(\mathrm{G}^{\prime \prime}\right)$ and loss $\left(\mathrm{G}^{\prime \prime}\right)$ modulus were decreased, as in almost all previously reported oleogels (Co and Marangoni 2012; Mezger 2014; Patel 2016; Mattice and Marangoni 2018). It is well known that oleogels retain their gelled consistency until the crossover point reached $\left(\mathrm{G}^{\prime}=\mathrm{G}^{\prime \prime}\right)$. Once crossover point was passed, the gel breaks down, and liquid oil starts to flow freely. The crossover points could be read from the temperature ramp graphs. The crossover points of the GMS-O, GMS/F68-O, and GMS/SP80-O samples were around 62,58 , and $60{ }^{\circ} \mathrm{C}$, respectively. Clearly, the addition of the amphiphiles reduced thermal stability a little. Similarly, flow point temperatures of around 55,56 , and $54^{\circ} \mathrm{C}$ were measured for the PGS-O, PGS/F68-O, and PGS/TW60-O samples.Further, it was clear that GMS oleogels had much better thermal stabilities than those of the PGS oleogels. In all GMS oleogels, the gelled states remains quite stable until around 50 ${ }^{\circ} \mathrm{C}$, and then slowly melts until around $60{ }^{\circ} \mathrm{C}$, but in PGS oleogels, the gel state become loose at around $30{ }^{\circ} \mathrm{C}$, and then get totally melted through $50-55{ }^{\circ} \mathrm{C}$. For all oleogels, the rheometer determined crossover points were higher than the DSC determined Tm values presented in Table 3. This phenomenon was also previously reported (Patel et al. 2013; Patel 2016; Mattice and Marangoni 2018). Since oscillatory rheometry determines not only temperature, but also the perceived resistance to flow, it could be quite possible that even after melting the fat crystals, some junction zones of the organogelators and co-gelators still exist to yield some viscosity. Further, the damping factor (loss factor) line (the tano line) was shown on the graphs. It indicates the phase transition from solid (gel state) to the liquid (sol state). Also, the crossing point of the damping factor line with the storage modulus $\left(\mathrm{G}^{\prime}\right)$ line could indicate the glass transition temperature. In all samples, the glass transition temperatures were lower than the flow point $\left(G^{\prime}=G^{\prime \prime}\right)$ temperatures. These temperature ramp test results give idea about until what temperatures the oleogels stays with their gelled structure. Moreover, during the gelation time measurements, it was clear that all oleogels prepared were thermo-reversible. This property could also be accepted as advantageous during heat processing of foods, in which oleogels were used in their formulae.

\section{Conclusion}

In this study, the effects of adding some selected amphiphiles on the structure and properties of oleogels 
prepared with GMS and PGS organogelators were investigated. In fact, there is a very limited number of studies with GMS oleogels, and none with PGS oleogels in literature, and hence information gained with this study may aid new applications of these oleogels. It was possible to decrease the additional levels of the organogelators with the addition of $3 \%(\mathrm{w} / \mathrm{w})$ selected amphiphiles (Pluronic F68, Span 80, and Tween 60). Amphiphile added oleogels showed larger crystal aggregates with softer structure, and lower melting points, but their oscillatory recovery abilities enhanced. They all were thermoreversible gels, with melting profiles similar to commercial margarine or solid lipids. Overall, these new oleogels would have some food applications with rheological, structural and thermal suitability.

\section{References}

Anonymous. (2020a). Glycerol monostearate. Retrieved from https://en.wikipedia.org/wiki/Glycerol_monostearate (Accessed in February 20, 2020).

Anonymous. (2020b). Product Specifications of Glycerol monostearate, purified. Retrieved from https://www.alfa.com/en/cat (Accessed in February 20, 2020)

Anonymous. (2020c). Product Data Sheet of Finamul PGS. Retrieved from https://www.fineorganics.com/additivesfor-food/emulsifiers/finamul-pge-2000 (Accessed in Fabruary 20, 2020).

Anonymous. (2020d). Poloxamer 188 solution. Retrieved from https://www.sigmaaldrich.com/catalog/product/sigma/p5556? (Accessed in Fabruary 20, 2020).

Anonymous. (2020e). Span @ 60. Retrieved from https://www.sigmaaldrich.com/catalog/product/SIGMA/S7010?lang=en\& NfD_BwE (Accessed in Fabruary 20, 2020).

Anonymous. (2020f). Tween 60. Retrieved from https://www.sigmaaldrich.com/catalog/substance/tween6012345900567811?1 (Accessed in Fabruary 20, 2020).

AOCS. (2012). AOCS Official Method Cj 2-95. X-ray diffraction analysis of fats. Official Methods and Recommended Practices of the AOCS, 6th Ed. 2011-2012 Methods and Additions and Revisions.

AOCS. (1998). Official Methods and Recommended Practices Vol I and II. American Oil Chemists' Society (4h ed.)

Bot, A., \& Flöter, E. (2018). Structuring edible oil phases with fatty acids and alcohols. In: Edible Oil Structuring: Concept, Methods and Applications, Ed. By Ashok R. Patel, pp. 95-105, Royal Soc. Chem., Chambridge, UK.

Cegla-Nemirovsky, Y., Aserin, A., \& Garti, N. (2015). Oleogels from glycerol-based lyotropic liquid crystals: phase diagrams and structural characterization. Journal of the American Oil Chemists' Society,92 : 439-447. https://doi.org/10.1007/s11746-015-2594-7

Chrysam, M.M. (1996). Margarines and Spreads. In: Bailey's Industrial Oil \& Fat Products, vol. 3, Ed. by Y.H. Hui, Wiley-Intersience Pub, New York, US.

Co, E.D., \& Marangoni, A.G. (2012). Organogels: an alternative edible oil-structuring method. Journal of the American Oil Chemists' Society,89 : 749-780. https://doi.org/10.1007/s11746-012-2049-3

Dassanayake, L.S.K., Kodali, D.R., \& Ueno, S. (2011). Formation of oleogels based on edible lipid materials. Current Opinion in Colloid \& Interface Science, 16 : 432-439. Doi:10.1016/j.cocis.2011.05.005

Mattice, K.D., \& Marangoni, A.G. (2018). New insights into wax crystal networks in oleogels. In: Edible Oil Structuring: Concept, Methods and Applications, Ed. by Ashok R. Patel, pp. 71-94, Royal Soc. Chem., Chambridge, UK.

Mezger, T. G. (2014). Applied Rheology. Anton Paar GmbH, Austria.

Minitab. (2010). Minitab Statistical Software (Version 16.1). Minitab, Inc., State College, Pennsylvania, US. 
Nakajima, N., \& Hamada, M. (2012). Synthesis of structurally well-defined polyglycelols and their fatty acid esters as new oil gelators. Journal of Synthetic Organic Chemistry Japan, 70(7) : 742-753. Doi: 10.5059/yukigoseikyokaishi.70.742

Patel, A. R. (2016). Understanding the oil-gelling properties of natural waxes. INFORM, 27(5) : 17-20. Doi: 10.21748/inform.06.2016.17

Patel, A. R., Schatteman, D., Vos, W. H. D., \& Dewettinck, K. (2013). Shellac as a natural material to structure a liquid oil-based thermo reversible soft matter system. RSC Advances, 3 : 5324-5327. https://doi.org/10.1039/C3RA40934A

Patel, A.R. (2018a). Oil structuring: concepts, overview and future perspectives. In: Edible Oil Structuring: Concept, Methods and Applications, Ed. by Ashok R. Patel, pp. 3-24, Royal Soc. Chem., Chambridge, UK.

Patel, A.R. (2018b). Edible Oil Stryucturing: Concepts, Methods and Applications . Royal Soc. Chemistry, Thomas Graham House, Cambridge, UK, 336 p.

Pomeranz, Y., \& Meloan, C.E. (1994). Food Analysis, Theory and Practice . Chapman \& Hall Publishing Co, New York

Sánchez, R., Franco, J.M., Delgado, M.A., Valencia, C., \& Gallegos, C. (2011). Rheology of oleogels based on sorbitan and glyceryl monostearates and vegetable oils for lubricating applications. Grasas y Aceites, 62(3) : 328-336. Doi: 10.3989/gya.113410

Singh, A., Auzanneau, F.I., \& Rogers, M.A. (2017). Advances in edible oleogel technology - a decade in review. Food Research International,97 : 307-317. doi: 10.1016/j.foodres.2017.04.022.

Uvanesh, K., Sagiri, S.S., Senthilguru, K., Pramanik, K., Banerjee, I., Arfat S., Al-Zahrani, M., \& Pal, K. (2016a). Effect of Span 60 on the microstructure, crystallization kinetics, and mechanical properties of stearic acid oleogels: an in-depth analysis. Journal of Food Science,81(2) : E380-E387. Doi: 10.1111/1750-3841.13170

Uvanesh, K., Sagiri, S.S., Banerjee, I., Shaikh, H., Pramanik, K., Anis, A., \& Pal, K. (2016b). Effect of Tween 20 on the properties of stearate oleogels: an in-depth analysis. Journal of the American Oil Chemists' Society, 93 : 711-719. Doi: 10.1007/s11746-016-2810-0

Wang, F.C., \& Marangoni, A.G. (2015). Internal and external factors affecting the stability of glycerol monostearate structured emulsions. RSC Advances, 5 : 93108-93116. https://doi.org/10.1039/C5RA18748F

Wang, F.C.,Challacombe, C., \& Marangoni, A.G. (2016). Effect of the addition of palm stearin and storage temperatures on the thermal properties of glycerol monostearate-structured emulsions. Food Research International, 79 : 29-32. Doi: 10.1016/j.foodres.2015.11.026

Yılmaz, E., Öğütcü, M., \& Güneser, O. (2015). Influence of storage on physico-chemical and volatile features of enriched and aromatized wax organogels. Journal of the American Oil Chemists' Society,92 : 1429-1443. https://doi.org/10.1007/s11746-015-2719-z

Yılmaz, E., \& Öğ̈utcü, M. (2014). Comparative analysis of olive oil organogels containing beeswax and sunflower wax with breakfast margarine. Journal of Food Science, 79(9) :

E1732-E1738. Doi: 10.1111/1750-3841.12561

\section{TABLES}

Table 1. The formulations used to prepare the glycerol monostearate (GMS) and polyglycerol stearate (PGS) oleogels

\begin{tabular}{llll}
\hline Sample Name & Sunflower Oil (\%) & Organogelator (10\%) & Amphiphile (3\%) \\
\hline GMS-O & 90 & Glycerol Monostearate (GMS) & - \\
GMS/F68-O & 87 & Glycerol Monostearate (GMS) & Pluronic F68
\end{tabular}




\begin{tabular}{llll}
\hline Sample Name $^{+}$ & Sunflower Oil (\%) & Organogelator (10\%) & Amphiphile (3\%) \\
\hline GMS/SP80-O & 87 & Glycerol Monostearate (GMS) & Span 80 \\
PGS-O & 90 & Polyglycerol Stearate (PGS) & - \\
PGS/F68-O & 87 & Polyglycerol Stearate (PGS) & Pluronic F68 \\
PGS/TW60-O & 87 & Polyglycerol Stearate (PGS) & Tween 60 \\
\hline
\end{tabular}

+ GMS-O: glycerol monostearate oleogel, GMS/F68-O: glycerol monostearate and pluronic F68 oleogel, GMS/SP80-O: glycerol monostearate and span 80 oleogel, PGS-O: polyglycerol stearate oleogel, PGS/F68-O: polyglycerol stearate and pluronic F68 oleogel, PGS/TW60-O: polyglycerol stearate and tween 60 oleogel

Table 2. Physico-chemical properties of the glycerol monostearate (GMS) and polyglycerol stearate (PGS) oleogels ${ }^{+}$

\begin{tabular}{llll}
\hline & Minimum Gelation Concentration $\left(\mathbf{C}^{*}, \boldsymbol{\%}\right)$ & Gelation Time (min) & Oil Binding Capacity (\%) \\
\hline GMS-O & $6.0 \pm 0.0^{\mathrm{a}++}$ & $3.3 \pm 0.6^{\mathrm{d}}$ & $100.0 \pm 0.01^{\mathrm{a}}$ \\
GMS/F68-O & $5.0 \pm 0.0^{\mathrm{b}}$ & $4.3 \pm 0.6^{\mathrm{c}}$ & $99.3 \pm 0.02^{\mathrm{a}}$ \\
GMS/SP80-O & $5.0 \pm 0.0^{\mathrm{b}}$ & $5.1 \pm 0.2^{\mathrm{b}}$ & $100.0 \pm 0.01^{\mathrm{a}}$ \\
PGS-O & $5.0 \pm 0.0^{\mathrm{b}}$ & $4.3 \pm 0.5^{\mathrm{c}}$ & $99.9 \pm 0.01^{\mathrm{a}}$ \\
PGS/F68-O & $4.0 \pm 0.0^{\mathrm{c}}$ & $6.3 \pm 0.6^{\mathrm{a}}$ & $99.6 \pm 0.02^{\mathrm{a}}$ \\
PGS/TW60-O & $4.0 \pm 0.0^{\mathrm{c}}$ & $5.3 \pm 0.6^{\mathrm{b}}$ & $99.9 \pm 0.01^{\mathrm{a}}$ \\
\hline
\end{tabular}

+After determination of the $\mathrm{C}^{*}$ values, the rest of the measurements were completed on the samples prepared by the formulae given in Table 1 .

${ }^{++}$Small letters within each column indicate significant differences among the oleogel samples for the mean +- SD values calculated from

four determinations by one-way analysis of variance and Tukey's test (p [?] 0.05)

Table 3. Thermal properties of the glycerol monostearate (GMS), polyglycerol stearate (PGS), and the oleogels prepared

\begin{tabular}{|c|c|c|c|}
\hline Crystalization & Crystalization & Crystalization & Crystalization \\
\hline & Onsetc $\left({ }^{\mathrm{o}} \mathrm{C}\right)$ & Peak $\left(\mathrm{Tc},{ }^{\mathrm{o}} \mathrm{C}\right)$ & $\Delta H_{\varsigma}(\Theta / \gamma)$ \\
\hline GMS-fr1 ${ }^{+}$GMS-fr2 & $15.12 \pm 0.2265 .46 \pm 0.19$ & $12.99 \pm 0.2162 .27 \pm 0.45$ & $-13.57 \pm 1.35-86.05 \pm 2.33$ \\
\hline PGS & $52.02 \pm 0.35$ & $49.53 \pm 0.15$ & $-90.35 \pm 141$ \\
\hline GMS-O-fr1 GMS-O-fr2 & $16.64 \pm 0.7254 .47 \pm 0.64$ & $14.16 \pm 0.1651 .06 \pm 0.05$ & $-2.56 \pm 0.01-7.67 \pm 0.02$ \\
\hline GMS/F68-O-fr1 GMS/F68-O-fr2 & $13.92 \pm 0.7853 .21 \pm 2.12$ & $11.85 \pm 1.0750 .85 \pm 2.25$ & $-2.27 \pm 1.09-9.36 \pm 3.42$ \\
\hline GMS/SP80-O-fr1 GMS/SP80-O-fr2 & $15.11 \pm 0.2052 .20 \pm 0.62$ & $12.59 \pm 0.0048 .58 \pm 0.47$ & $-1.67 \pm 0.11-7.92 \pm 1.64$ \\
\hline PGS-O & $33.63 \pm 0.44$ & $31.83 \pm 0.37$ & $-2.90 \pm 0.55$ \\
\hline PGS/F68-O & $34.77 \pm 1.73$ & $32.68 \pm 2.02$ & $-4.92 \pm 0.90$ \\
\hline PGS/TW60-O & $30.58 \pm 0.13$ & $29.07 \pm 0.21$ & $-3.11 \pm 0.25$ \\
\hline
\end{tabular}

+fr1: fraction one; fr2: fraction two

\section{Figure Legends}

Fig. 1. The oleogel samples prepared with glycerol monostearate (GMS), polyglycerol stearate (PGS) and some amphiphiles combinations. 
Fig. 2. The polirized light microscopy images of the oleogels with 40x magnification (a-GMS-O, bGMS/F68-O, c-GMS/SP80-O, d-PGS-O, e-PGS/ F68-O, f-PGS/TW60-O).

Fig. 3. The X-ray diffraction patterns of the oleogel samples prepared.

Fig. 4. The amplitude sweep test results of the oleogel samples prepared.

Fig. 5. The frequency sweep test results of the oleogel samples prepared.

Fig. 6. The time sweep test results of the oleogel samples prepared.

Fig. 7. The temperature ramp test results of the oleogel samples prepared.

\section{Hosted file}

Figure 1.docx available at https://authorea.com/users/315025/articles/445354-comparison-of-theglycerol-monostearate-and-polyglycerol-stearate-oleogels-effects-of-amphiphile-addition

\section{Hosted file}

Figure 2.docx available at https://authorea.com/users/315025/articles/445354-comparison-of-theglycerol-monostearate-and-polyglycerol-stearate-oleogels-effects-of-amphiphile-addition

\section{Hosted file}

Figure 3.docx available at https://authorea.com/users/315025/articles/445354-comparison-of-theglycerol-monostearate-and-polyglycerol-stearate-oleogels-effects-of-amphiphile-addition

\section{Hosted file}

Figure 4.docx available at https://authorea.com/users/315025/articles/445354-comparison-of-theglycerol-monostearate-and-polyglycerol-stearate-oleogels-effects-of-amphiphile-addition

\section{Hosted file}

Figure 5.docx available at https://authorea.com/users/315025/articles/445354-comparison-of-theglycerol-monostearate-and-polyglycerol-stearate-oleogels-effects-of-amphiphile-addition

\section{Hosted file}

Figure 6.docx available at https://authorea.com/users/315025/articles/445354-comparison-of-theglycerol-monostearate-and-polyglycerol-stearate-oleogels-effects-of-amphiphile-addition

\section{Hosted file}

Figure 7.docx available at https://authorea.com/users/315025/articles/445354-comparison-of-theglycerol-monostearate-and-polyglycerol-stearate-oleogels-effects-of-amphiphile-addition 